\title{
Dinámica sociecológica de una comunidad de campesinos de la vereda San Luis, Barrancabermeja
}

\author{
Jesús Quintero-Cardozo ${ }^{1}$ \\ Adriana Patiño-Núñez ${ }^{2}$ \\ Henrique Riva ${ }^{3}$ \\ Kelly Cristina Torres-Angulo ${ }^{4}$
}

Fecha de recepción: 1 de marzo de 2019

Fecha de aceptación: 6 de julio de 2019

\section{Resumen}

Este artículo analiza la dinámica socioecológica de comunidades campesinas asentadas en la vereda de San Luis del municipio de Barrancabermeja, Santander, Colombia. Se analizaron los patrones de comportamiento de las comunidades, el entorno natural y la incidencia de las instituciones que hacen parte del sistema. Utilizando las herramientas conceptuales y metodológicas del marco de análisis y desarrollo institucional (IAD), se pretende ubicar el conjunto de reglas formales e informales que influyen en la relación hombre/naturaleza y develar arreglos institucionales que permitan identificar conflictos con especies animales (particularmente felinos) y estrategias de conservación. Se concluye que las principales fallas institucionales, están dadas por los conflictos por el uso de recursos naturales entre las diferentes denominaciones que hacen parte del territorio (campesinos antiguos, desplazados y academia). Dicha divergencia es potenciada por la desigualdad social e inequidad de apoyo por parte del gobierno, que también contribuye para que los campesinos desplazados tengan un uso mucho más enfocado en alta productividad, en contraste con el uso de los campesinos antiguos que muestra ser de subsistencia o para comercio en pequeñas cantidades. Se hace necesario fortalecer la institucionalidad rural y poner las reglas formales en acción para lograr sinergias que permitan aumentar la eficiencia y eficacia en la utilización de los recursos naturales para lograr mejorar la relación entre hombre y naturaleza.

Palabras clave: conflicto cultural; conservación ambiental; desarrollo participativo; organización.

\footnotetext{
1 M. Sc. Instituto Universitario de la Paz - UNIPAZ (Santander, Colombia). jesus.quintero@unipaz.edu.co. ORCID: 0000-0003-2419-4746.

2 M. Sc. Pontificia Universidad Javeriana (Bogotá-Distrito Capital, Colombia). am patinon@javeriana.edu.co. ORCID: 0000-0002-7594-1097.

${ }^{3}$ Fundación Botánica y Zoológica de Barranquilla (Barranquilla, Colombia). ORCID: 0000-0001-7584-7706.

${ }^{4}$ Instituto Universitario de la Paz - UNIPAZ (Santander, Colombia).
} 


\section{Socioecological Dynamic of a Rural Community at San Luis, Barrancabermeja}

\section{Abstract}

This article analyzes the socio-ecological dynamics of local communities settled in the village of San Luis in the municipality of Barrancabermeja, Santander, Colombia. We analyze patterns of behavior of communities, the natural environment and the impact of institutions that are a part of the system. Using the conceptual and methodological tools of the institutional analysis and development framework (IAD), it is intended to locate the set of formal and informal rules that influence the man / nature relationship and unveil institutional arrangements to identify conflicts with animal species (specially felines) and conservation strategies. The conclusion is that most institutional failures are given by the conflicts between the different groups that are part of the territory (old communities, new communities of people displaced by violence and academia). This divergence is fueled by social inequality and disparity of support from the government that also encourage displaced communities to use much more resources focused on high productivity, on the other hand, old communities have a resource use focused on subsistence or low-scale trade. It is necessary to strengthen rural institutions and put formal rules in action to achieve synergies that increase the efficiency in the use of natural resources to improve the relationship between man and nature.

Keywords: cultural conflict; environmental conservation; organization; participatory development.

\section{Para citar este artículo:}

Quintero-Cardozo, J., Patiño-Núñez, A., Riva, H., \& Torres-Angulo, K. C. (2019). Dinámica sociecológica de una comunidad de campesinos de la vereda San Luis, Barrancabermeja. Ciencia y Agricultura, 16(3), 61-73. https://doi.org/10.19053/01228420.v16.n3.2019.9889.

Esta obra está bajo licencia internacional Creative Commons Reconocimiento 4.0

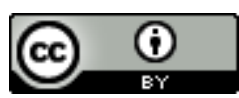




\section{INTRODUCCIÓN}

Las actividades agrícolas constituyen una modificación del ecosistema para la satisfacción de las necesidades básicas de alimento, fibras y otros materiales (Cleves, Toro, Martínez, \& León, 2017), llevando a que grandes áreas de bosques tropicales nativos hayan sido reducidas a pequeños fragmentos naturales inmersos en una matriz agrícola y de pastoreo, generando importantes preocupaciones ecológicas debido a los impactos producidos (Etter, McAlpine, Pullar, \& Possingham, 2005).

Entre ellos, se ha reconocido la pérdida de biodiversidad con la reducción y fragmentación del hábitat, contaminación ambiental (Etter et al., 2005; Muñoz \& Muñoz, 2016), o la generación de conflictos entre la fauna silvestre propia de los ecosistemas intervenidos que entra a cohabitar con el agroecosistema, entre ellos, el consumo y destrucción de cultivos y alimentos almacenados, la depredación de animales domésticos o la transmisión de enfermedades a humanos u otros animales (Muñoz \& Muñoz, 2016).

El conocimiento de cómo se emplean los recursos y los conflictos ambientales generados a partir de dicho uso, conforman una línea base para entender la relación sociedad y medio ambiente y con ello definir criterios para diseñar formas de manejo de los recursos, que integren los sistemas productivos y la conservación del medio ambiente (Ojasti \& Dallmeier, 2000).

El análisis de estos agroecosistemas implica reconocer que en ellos persisten múltiples interacciones de tipo biofísico, social, económico, político y tecnológico (Cleves et al. 2017), dentro de las cuales operan normas (instituciones) determinantes en las decisiones e interacciones humanas.

El Marco de Análisis y Desarrollo Institucional (IAD) es una herramienta teórica y metodológica que permite analizar contextos donde el diseño y operación instituciones afectan el comportamiento o toma de decisiones del ser humano, que en este caso serían determinantes en la interacción entre la sociedad y el uso de los recursos (Ángel, 2002; Benedetti, 2017).

Las instituciones plantean las reglas del juego (conjunto de prescripciones y limitaciones) que los seres humanos usan para organizar todo tipo de interacciones repetitivas y estructuradas, incluyendo reglas, normas y estrategias compartidas (Ostrom, 2005). Puede ser de carácter formal, como las leyes, o informal, como las reglas de uso relacionadas con costumbres o modos de proceder (Ostrom, 1991; North, 1990 citados por Benedetti, 2017).

Su presencia o ausencia generan oportunidades o restricciones que afectan las acciones o decisiones de las personas que interactúan dentro de situaciones estructuradas (Ostrom, 2005). En este sentido, la aplicación del IAD permite identificar en una situación de interacción social con los recursos, que actores están 
involucrados, qué tipos de instituciones están rigiendo esta interacción y cuáles son las respuestas de los actores a estas normas de uso de los recursos comunes.

Este trabajo se centra en identificar cuáles son estas actividades de uso de los recursos realizados por la sociedad en un relicto de bosque húmedo tropical de la vereda San Luis (Barrancabermeja, Santander, Colombia); qué tipos de efectos está teniendo tanto para el ecosistema como para la comunidad y posibles conflictos, dando mayor énfasis al conflicto con grandes felinos usando el marco IAD.

El análisis de las reglas y las consecuencias (sección en gris, Figura 1) que rigen estos procesos de interacción y las posibles formas de mejoramiento de estos sistemas de gobernanza corresponden a aspectos siguientes a desarrollar para culminar con la aplicación de IAD, para finalmente desarrollar una estrategia que permita la conservación del bosque húmedo tropical y su relación con la comunidad.

\section{Metodología}

La investigación se realizó en la Vereda San Luis, del municipio de Barrancabermeja (Santander, Colombia), al margen oriental del río Magdalena, a $75 \mathrm{msnm}$, en donde se actualmente encuentra un relicto de bosque húmedo tropical ubicado en instalaciones del Instituto Universitario de la Paz - UNIPAZ y una comunidad de campesinos.

En esta región la comunidad está conformada por pequeños campesinos minifundistas, ganaderos (ganado bovino y bufalino), agricultores (caucho y palma), y el Instituto Universitario de la Paz - UNIPAZ.

Para comprender el contexto socioecosistémico donde está inserida la comunidad de la vereda y sus diferentes actores, este trabajo presenta un enfoque epistemológico, basado en la percepción de la comunidad acerca de los tipos de uso y conflictos entorno a uso del bosque, información recopilada a través de entrevistas semiestructuradas y complementada con la revisión de la caracterización a priori realizada por Acuña (2017).

Como criterios de inclusión de las personas entrevistadas se consideró que estas llevaran tiempo de permanencia en la región, y que sus actividades de subsistencia fueran realzadas principalmente en torno al uso de los recursos del bosque y sus alrededores.

La información primaria fue consultada con cinco representantes de la comunidad: el presidente de la Junta Comunal de la Vereda de San Luis, el vicepresidente de la Asociación de Desplazados por el Derecho a la Vida, una campesina moradora de la vereda y delegados de la academia como el Coordinador del Programa de Monitoreo de Fauna Silvestre en el Instituto Universitario de la Paz (UNIPAZ) y la Vicerrectora de UNIPAZ. 
Las entrevistas tuvieron como base cuatro temas principales: tiempo de ocupación de la vereda; fuentes de ingresos; usos del bosque y recursos ambientales comunes; conflictos entre actores y con la fauna relacionados al uso de recursos comunes. La formulación de cada pregunta y su desarrollo fue adaptada en cada entrevista considerando el nivel social, cargo o profesión ocupada por cada actor.

La información generada fue utilizada para aplicar el marco de IAD desarrollado por Ostrom (2005) con base en los elementos que exige un análisis institucionalista: variables exógenas, arena de acción, interacciones, resultados y criterios de evaluación (Figura 1).

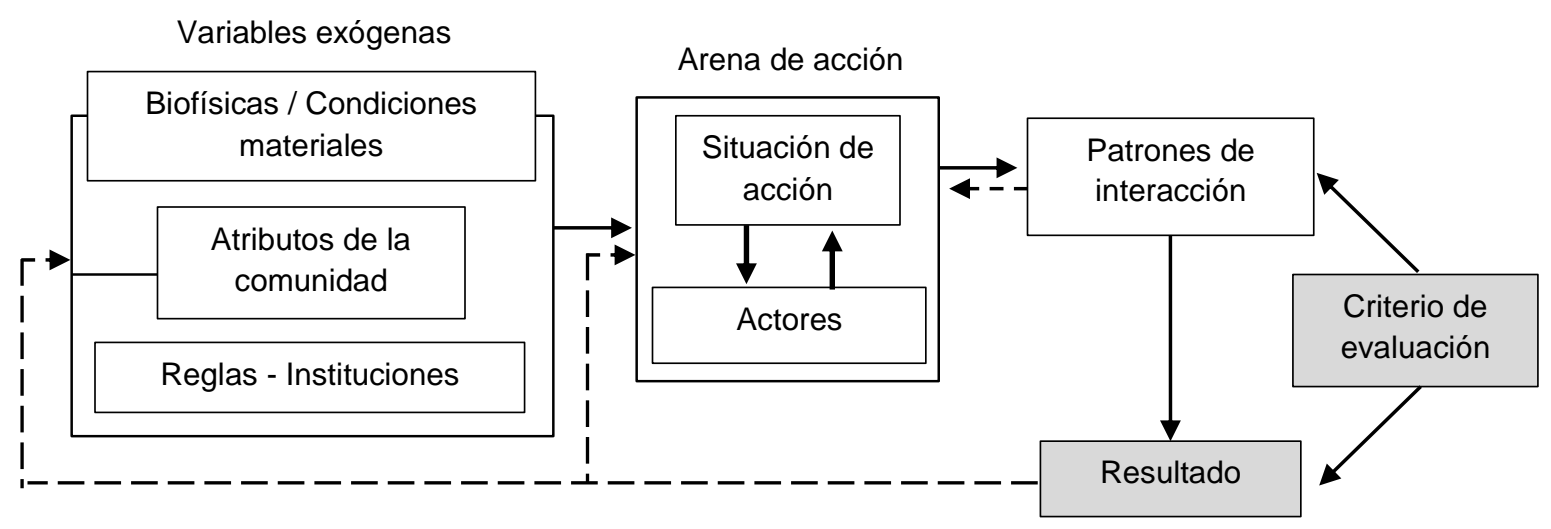

Fig. 1. Marco de análisis y desarrollo institucional. Adaptado de Ostrom, Gardner y Walker (1994) y Ostrom (2005).

Las variables exógenas corresponden a las "condiciones materiales o biofísicas», los «atributos de la comunidad» y las «reglas - instituciones» que influyen sobre el escenario de acción (Ostrom, 2005).

El ambiente biofísico se define por la configuración estructural del área que puede limitar las acciones posibles y los resultados que pueden ser alcanzados, teniendo en cuenta la disponibilidad de recursos. Las características de la comunidad son los atributos de ésta, que influencian la toma de decisiones, similitudes y diferencias entre los actores y sus opiniones, incluyendo la situación socioeconómica. Las reglas o instituciones son definidas por instituciones o lineamientos que guían o modulan las relaciones e influencian las decisiones de los actores, definen qué acción es permitida, requerida o prohibida, estas, pueden ser escritas en leyes oficiales, pero también pueden ser informales, cómo acuerdos verbales, y constituye una de las principales áreas de estudio de IAD, requiriendo un amplio conocimiento de la comunidad, principalmente para identificar instituciones informales (Nigussie et al., 2017; Ostrom, Gardner, \& Walker, 1994; Ostrom, 2005).

La «arena de acción» (o área de acción) es el núcleo del método de IAD, compuesta por dos elementos: la «situación de acción» y los «actores» (Ostrom, 1994), ambos vitales para entender la toma de decisiones en relación al uso de recursos comunes. 
La situación de acción se define por ser el espacio o actividad social donde interactúan los actores, es decir, las situaciones en que se toman decisiones. Los participantes o actores son aquellos que interactúan en la comunidad, que participan en la situación y tienen poder de decisión sobre el uso de los recursos (Nigussie et al., 2017; Ostrom, 2005; Benedetti, 2017).

Los «patrones de interacción» son flujos de información en la comunidad y condiciones de interacción entre los actores. También involucra el método o espacio de aprendizaje de nuevas prácticas o informaciones y es influenciado y al tiempo sufre influencia de la arena de acción.

El «criterio de evaluación» son las características medibles para evaluar los resultados de la intervención, patrones de interacción, influencia de las instituciones y resultados (Ostrom, 2005)

Finalmente, el «resultado» es el efecto de las interacciones y del contexto socioeconómico de la arena de acción de los actores involucrados (Nigussie et al., 2017; Ostrom et al., 1994; Ostrom, 2005).

\section{ResULtADOS}

\section{A. Usos y servicios de los recursos naturales en torno al bosque húmedo tropical}

Según el trabajo realizado por Acuña (2017), en donde se consultó la principal actividad económica realizada por las comunidades de la vereda (familias, personas, etc), en el área se encuentran actividades productivas como parcelas de explotación de pancoger (33\%), ganadería (19\%), jornaleros en actividades relacionadas con el sector hidrocarburo y forestal (16\%), transporte informal (17\%), comercio formal $(7 \%)$, obreros de proyectos viales (4\%) y pesca artesanal $(4 \%)$.

Adicionalmente, con la información reportada en las entrevistas se identificaron otros usos relacionados con la extracción de leña y elaboración de carbón vegetal, obtención de madera para construcción de viviendas y algunas fibras para techos, extracción de madera y productos no maderables del bosque para uso doméstico y comercialización, comercio ilegal de fauna silvestre y caza de subsistencia, uso de material foliar para actividades culturales y comerciales, esparcimiento, paisaje y atractivos naturales, presencia y establecimiento de comunidades de pensamiento y espiritualidad y actividades de investigación en torno a la fauna y la flora. Estos usos no han sido considerados para evaluar su grado de impacto y sobre los cuales existen reglas de uso.

El entorno natural en la región provee servicios ecosistémicos a las comunidades locales, por ejemplo: la acumulación de germoplasma dentro de los ecosistemas de bosque, acumulación de aguas de escorrentía (bajos inundables), estabilización de rondas hídricas, presencia de relictos boscosos que controlan deslizamiento y evitan arrastre de suelos, presencia de relictos boscoso como sumideros de carbono, regulación de temperaturas, precipitación y humedad, corredor biológico y 
hábitat de especies sombrilla (Panthera onca) y endémicas (Tapura bullata), aporte de biomasa, ciclo de nutrientes, consolidación de horizontes, ciclo de materia orgánica.

\section{B. Conflictos en la comunidad relacionados con el uso de los recursos asociados al bosque húmedo tropical}

El uso de los recursos ha repercutido en la transformación del paisaje del bosque húmedo tropical en matrices agropecuarias en la Vereda de San Luis, que conllevan a procesos de fragmentación, reducción de hábitat y pérdida de biodiversidad del bosque húmedo tropical. Situación de pérdida de hábitat principalmente evidenciada en la última década de más de 130.000 ha de humedales; ha incrementado en más de cuatro millones de metro cuadrados los cultivos de Palma de aceite y en 20.000 ha las pasturas. Como resultado, más del $34 \%$ del suelo presenta usos inadecuados y otro $20 \%$ se considera subutilizado (SILAP, 2016); lo que implica que al menos el $54 \%$ del territorio se encuentra mediado por conflictos socioambientales.

Este escenario, aunado a la dependencia económica de una industria petrolera en crisis (aproximadamente $70 \%$ de la economía depende del petróleo), ha incrementado la presión antrópica sobre los ecosistemas naturales, mediante el cambio de uso del suelo y la sobreexplotación del bosque y los humedales. En este contexto se mueve la fauna asociada al bosque, entre los que se encuentran los grandes mamíferos de la región que sufren de manera directa la disminución de los parches, la fragmentación del ecosistema y con ello la pérdida de conectividad (corredores biológicos), además de la extracción de productos vegetales y animales. Esta pérdida de hábitat ha permitido un conflicto entre los seres humanos y animales, en particular; grandes felinos, en aspectos relacionados con la supervivencia y espacio geográfico (territorio).

Las respuestas de los diferentes actores a las entrevistas fueron recopiladas en un video retractando el contexto de la comunidad de la Vereda de San Luis. Algunas respuestas relevantes con relación a la relación de la comunidad con los jaguares en la región están transcritas en la Tabla 1. La comunidad mostró una discrepancia en considerar o no a los felinos como una amenaza para las actividades de ganadería y también cuanto al impacto de la pecuaria por la contaminación del recurso hídrico.

Tabla 1. Declaraciones de diferentes actores de la comunidad de la Vereda de San Luis en la luz del conflicto con grandes felinos.

\begin{tabular}{|c|l|}
\hline Actor & \multicolumn{1}{|c|}{ Opinión recopilada } \\
\hline $\begin{array}{c}\text { Presidente de la Junta } \\
\text { Comunal de la Vereda } \\
\text { de San Luis }\end{array}$ & $\begin{array}{l}\text { "En estos momentos, no hemos tenido problemas con estos animales } \\
\text { [jaguares]. Anteriormente si, hace muchísimos años en el año 60 o 70, } \\
\text { llegaban muchos animales de estos y nos hacían daño... Y cuando eso } \\
\text { pues no había ni ley ni nada entonces todo el mundo dale chumbimba... } \\
\text { Todos estos animales fueron desapareciendo. Los dos animales que hay } \\
\text { ahí le tienen más miedo a la gente." }\end{array}$ \\
\hline $\begin{array}{c}\text { Vicepresidente de la } \\
\text { Asociación de } \\
\text { Desplazados por el } \\
\text { Derecho a la Vida }\end{array}$ & $\begin{array}{l}\text { los animales... la pantera, el puma ese, el tigre, nos está perjudicando } \\
\text { mucho y muchos más animales." }\end{array}$ \\
\hline
\end{tabular}

Ciencia y Agricultura (Cien. Agri.) Vol. 16 (3). L-ISSN 0122-8420. eISSN 2539-0899.

Septiembre-Diciembre 2019, pp. 61-73. Tunja (Boyacá) - Colombia. DOI: https://doi.org/10.19053/01228420.v16.n3.2019.9889 


\begin{tabular}{|c|l|}
\hline Actor & \multicolumn{1}{|c|}{ Opinión recopilada } \\
\hline $\begin{array}{c}\text { Docente y Coordinador } \\
\text { del Programa de } \\
\text { Monitoreo de Fauna } \\
\text { Silvestre en UNIPAZ }\end{array}$ & $\begin{array}{l}\text { "Entonces en la medida que nosotros podamos armonizar esa relación a } \\
\text { través del diseño de sistemas productivos que sean sostenibles...Que } \\
\text { podamos medir indicadores de biodiversidad dentro de mi ganadería. } \\
\text { Que yo use la biodiversidad, pero dejo que ella también se recupere para } \\
\text { también poder mantener una rotación dentro de mi producción." }\end{array}$ \\
\hline Vicerrectora de UNIPAZ & $\begin{array}{l}\text { "No podríamos desligar esa relación que existe de la comunidad con la } \\
\text { misma academia...porque todos estamos inmersos en un mismo } \\
\text { entorno... A todos nos está afectando lo que pasa en ese ecosistema. Al } \\
\text { establecer una relación tan directa como la que vamos a plantear, } \\
\text { estamos abordando un poco la problemática que tiene la comunidad, le } \\
\text { estamos posiblemente brindando unas alternativas desde el punto de } \\
\text { vista productivos...y también vamos a estar brindando unas alternativas } \\
\text { a unos problemas de saneamiento que existen en estas comunidades." }\end{array}$ \\
\hline $\begin{array}{c}\text { Campesina moradora } \\
\text { de la Vereda de San }\end{array}$ & $\begin{array}{l}\text { "Bufaler está dañando los pantanos, los potreros. De las bufaleras salen } \\
\text { muchas aguas pichas. Se dañan los pastos al marchetar... cuando crece, } \\
\text { Luis } \\
\text { eso se estanca y al que se va mermando bota fétida el agua. Eso también } \\
\text { cae al Zarzal y esto molesta como tanto a las personas que consumen el } \\
\text { agua como a nosotros que vivimos del pescado...para nosotros comer... } \\
\text { Y a veces el pescado se va, se desaparece o se muere... hemos visto } \\
\text { blanquillos boyados, supremamente dañados por las aguas" }\end{array}$ \\
\hline
\end{tabular}

\section{Marco IAD entorno a la presencia de grandes felinos circundantes a zonas de uso del Bosque Húmedo Tropical}

La información obtenida fue evaluada en el marco de IAD con el objetivo de comprender el contexto de la comunidad y la posición de los diferentes actores con relación a la presencia de grandes felinos en la región (Figura 2).

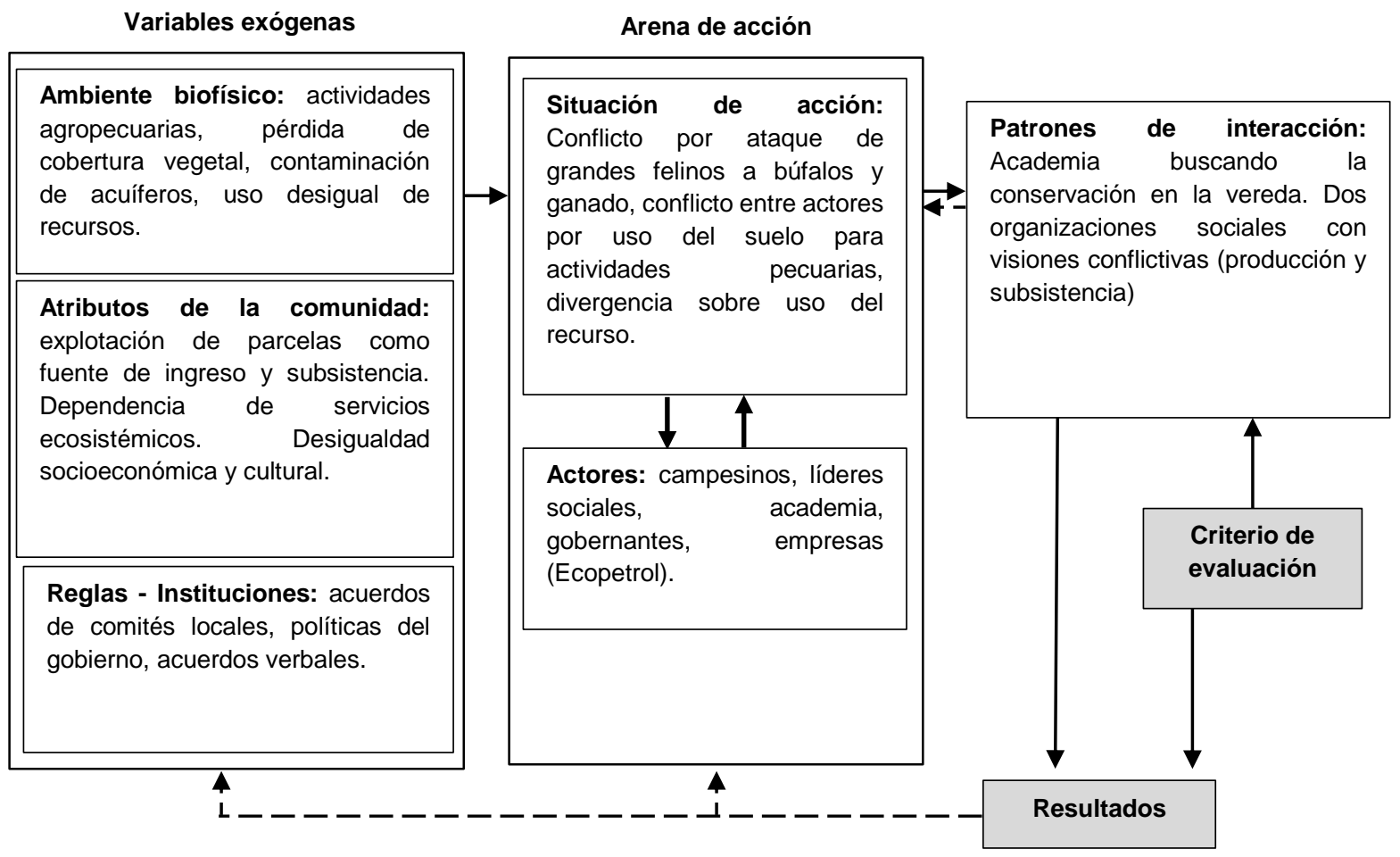

Fig. 2. Marco de Análisis y Desarrollo Institucional de la Vereda de San Luis a la luz del conflicto con grandes felinos. 
Considerando que el presente estudio fue desarrollado a corto plazo, la información generada, constituye una visión general y superficial de las instituciones que rigen a la comunidad, y de la influencia de otros actores como el gobierno regional, nacional, por ello no se incluyó en el diagrama el criterio de evaluación o el resultado.

Las partes involucradas en las actividades que pueden llegar a tener un conflicto con los grandes felinos o que tienen influencia bajo sus instituciones corresponden a: moradores de la vereda (campesinos), personal del gobierno comunitario (líderes sociales), investigadores de UNIPAZ (Academia), gobernantes y empresas (Ecopetrol).

Los moradores de la región que hacen uso económico o cultural de los recursos asociados al bosque húmedo tropical y en entorno a este hacen parte de 41 familias. De estas, el $88 \%$ son de origen rural y el $12 \%$ de origen urbano. De estas familias el $41,46 \%$ habitan el sitio desde hace 20 a 30 años, el $21,9 \%$ desde hace 10 a 20 años y el $36,58 \%$ se encuentra en el sitio desde hace menos de 10 años. Este último grupo hace parte de familias víctimas del conflicto armado reubicadas en la vereda por parte del programa nacional de restitución de tierras. En relación a la edad de las personas, se encontró que la mayoría de los habitantes son adultos entre los 18 a 59 años.

El personal de gobierno comunitario está conformado por la Junta Comunal a la cual hacen parte los habitantes con arraigo territorial, y la Asociación de Desplazados por el Derecho a la Vida conformado por las familias partícipes del programa nacional de restitución de tierras.

UNIPAZ está conformado por investigadores multidisciplinarios que realizan actividades de capacitación con la comunidad relacionadas con el uso sustentable de los recursos y jornadas de monitoreo dentro del relicto de bosque húmedo tropical para identificar su biodiversidad y el estado de conservación.

Dentro de los gobernantes es de interés la Unidad Administrativa Especial de Gestión de Restitución de Tierras Despojadas, adscrita al Ministerio de Agricultura y Desarrollo Rural como órgano administrativo del Gobierno Nacional encargada de coordinar la compensación de las víctimas del conflicto armado, y las empresas que realizan sus actividades económicas en la región, como el caso de Ecopetrol.

Estos actores están inmersos en un ambiente ecosistémico caracterizado por presentar relictos de bosque húmedo tropical acompañado de unas grandes fuentes hídricas como la Ciénega de San Silvestre, Sábalo y Zarzal, rodeado de un paisaje agropecuario principalmente aprovechado para cultivos de pancoger y ganadería y cementeras, que han sido asociadas a procesos de contaminación del agua.

En ese contexto, se observó una divergencia de los campesinos de arraigo territorial (Señora Ofelia y Señor Adalberto) con los campesinos desplazados por la violencia 
(Señor Isaías) en relación al impacto generado por los grandes felinos en la región. El primer grupo considera que existe una reducción del número poblacional de estas especies, las cuales no se aproximan a las áreas usadas para el cuidado de animales domésticos, por el contrario, el segundo grupo ve en los grandes felinos una amenaza para sus actividades económicas.

Dicha divergencia podría estar asociada a las actividades económicas realizadas por cada grupo y la diferencia en fuentes de ingreso entre los actores. El grupo de familias participes del programa de restitución de tierras cuenta con el apoyo económico y técnico del estado para desarrollar la ganadería extensiva, actividades bufaleras y otras actividades económicas con producción a ingresos a mediana y gran escala. En contraposición, las familias de arraigo territorial no cuentan con estos apoyos y ejercen la ganadería en pequeñas áreas de lotes familiares, su producción pecuaria se centra en la subsistencia y comercio a baja escala. Por esta razón, es posible que los eventos de conflicto con grandes felinos sean más frecuentes durante las actividades pecuarias a gran escala generando mayor insatisfacción para las familias del programa de restitución de tierras. Por otro lado, para campesinos con otras fuentes de ingreso, como cultivos de pancoger $(33 \%)$ o pesca artesanal (4\%), por ejemplo, la Señora Ofelia, el conflicto con los grandes felinos es inexistente y los animales no son mencionados más allá de los registros observados de sus rastros. No entanto, este grupo de campesinos tiene fuertes opiniones contrarias a la creación de bufaleras debido a que dicha actividad daña los pastos y contamina las fuentes de agua, imposibilitando su uso y actividades pesqueras (ejemplo en cuadro 1).

En Colombia existen seis especies de felinos silvestres. Las amenazas para todas las especies son la pérdida, transformación y fragmentación del hábitat producto del avance de la frontera agropecuaria, construcción de carreteras y poblados y la cacería retaliativa especialmente de jaguares y pumas por ataques a animales domésticos. Haciendo de este último el más importante componente del conflicto humano-felinos (Payan \& Soto, 2012). Ante la percepción de amenazas por parte de la comunidad, durante varios años fue común que se ejerciera la cacería (ejemplo en tabla 1), sin embargo, con la reglamentación y sanciones producto del Código Nacional de los Recursos Naturales y de Protección del Medio Ambiente (Decreto-Ley 2811 de 1974), se influyó para que este tipo de actividades disminuyeran, normas que están actualmente presentes entre los campesinos y que influyen en la toma de decisiones ante el este conflicto.

Ante las pérdidas económicas que representa para la ganadería la pérdida de bovinos y búfalos, es posible que puedan incentivarse posibles retaliaciones producto del ataque por parte de los felinos, por cuanto se requerirá evaluar con mayor detalle si existe o no cacería hacia jaguares o pumas, de otra manera, aunque la comunidad de arraigo cultural indica que la cacería ya no se realiza, es posible que dichas familias tuvieran la misma reacción si la situación de conflicto aún fuera vigente con su fuente principal de ingreso económico. 
Por otro lado, la visión de la academia tiene un enfoque conservacionista, muy diferente de lo observado en ambos grupos de campesinos, buscar un equilibrio en los objetivos y demandas de los tres grupos para los recursos de la región, parece ser el principal reto (Patrones de interacción, Figura 2). La influencia de UNIPAZ, bajo unos objetivos de conservación, promueve el desarrollo de proyectos de extensión con los campesinos de arraigo territorial y ha avanzado en ofrecerles alternativas de ingreso, y la búsqueda del cambio de percepción hacia los recursos naturales. Incentivando también el reconocimiento de la biodiversidad de la región y su conservación. Estas actividades de apropiación social del conocimiento son, en gran forma, mecanismos por los cuales la institucionalidad de la academia influye en las decisiones de los moradores en torno a los recursos. Sin embargo, bajo condiciones de marginalidad ecológica, social y político-económica, la influencia de la institucionalidad de la academia es baja. Adicionalmente, la discusión de la academia con el grupo de desplazados todavía no ha empezado.

\section{Discusión y CONCLUSIONES}

En relación al origen y tiempo de permanencia en la región, pueden encontrarse variaciones en el sentido de pertenencia y el arraigo cultural, que pueden ser influyentes en los tipos de decisiones y acciones que realizan hacia el uso de los recursos. Hay divergencias de posiciones y opiniones en relación a los tres actores evaluados (campesinos antiguos, desplazados y academia) con un conflicto identificado entre los dos grupos de campesinos en relación al uso del suelo. Dicha divergencia es potenciada por la desigualdad social e inequidad de apoyo por parte del gobierno que también contribuye para que los campesinos desplazados tengan un uso mucho más enfocado en alta productividad. Las diferencias entre los actores llevan a una visión que también es diversa en relación a los grandes felinos en la región y a la contaminación del agua causada por las actividades pecuarias. Ambos conflictos demandan más estudios para que puedan ser comprendidos a fondo y se puedan buscar soluciones.

Uno de los principales retos para la compresión de la interacción hombre-felinos es el efecto del apoyo desigual ofrecido por el gobierno a los dos grupos de campesinos, con incentivos económicos significativos y apoyo técnico para el establecimiento de las familias desplazadas, mientras que las familias originalmente habitantes de la región no reciben la misma atención. Dicha política pública contribuye para generar conflicto entre los dos grupos, dificulta la comunicación, tiene consecuencias sobre su visión sobre los efectos de las actividades productivas desarrolladas y el uso de recursos comunes.

\section{AgradeCIMIENTOS}

Los autores agradecen al Instituto Universitario de la Paz - UNIPAZ, a la Pontificia Universidad Javeriana, al doctor Cesar Enrique Ortiz Guerrero y a los pobladores de la vereda San Luis del municipio de Barrancabermeja por permitir seguir construyendo ciencia e investigación para el desarrollo rural. En particular, conocer las dinámicas de desarrollo para la construcción de un mejor entorno. De igual 
manera expresan que esta investigación aporta al desarrollo de los productos del proyecto denominado "Diseño de sistemas productivos sostenibles como estrategia de conservación de paisajes rurales sostenibles, Barrancabermeja, Santander".

\section{REFERENCIAS}

Acuña, S. (2017). Caracterización socioambiental y productiva de la vereda San Luis. Instituto Universitario de la Paz (UNIPAZ). Barrancabermeja, Colombia.

Ángel Lara, H. (2002). El gobierno de los bienes comunes: la evolución de las instituciones de acción colectiva, Región y sociedad. 14(24), 263-269. https://doi.org/10.22201/cimsur.18704115e.2010.10.155.

Benedetti, A. (2017). El marco de análisis y desarrollo institucional (IAD), una herramienta de análisis de políticas públicas. Estudio del caso Agro Ingreso Seguro (AIS). Estudios Políticos, 50, 138-158. https://doi.org/10.17533/udea.espo.n50a08.

Cleves-Leguízamo, J. A., Toro-Calderón, J., Martínez-Bernal, L. F., \& León-Sicard, T. (2017). La Estructura Agroecológica Principal (EAP): novedosa herramienta para planeación del uso de la tierra en agroecosistemas. Revista Colombiana de Ciencias Hortícolas, 11(2), 441-449. https://doi.org/10.17584/rcch.2017v11i2.7350.

Etter, A., McAlpine, C., Pullar, D., \& Possingham, H. (2005). Modeling the age of tropical moist forest fragments in heavily-cleared lowland landscapes of Colombia. Forest Ecology and Management, 208 (1-3), 249-260. https://doi.org/10.1016/j.foreco.2004.12.008.

Laurance, W. F., \& Ferreira, L. V. (1997). Effects of forest fragmentation on mortality and damage of selected trees in central Amazonia. Conserv. Biol, 11, 797-801. https://doi.org/10.1046/j.1523-1739.1997.96167.x.

Laurance, W. F., Ferreira, L. V., Rankin-de Merona, J., \& Laurance, S. G. (1998). Rain forest fragmentation and the dynamics of Amazonian tree communities. Ecology, 79, 2032-2040. https://doi.org/10.1890/00129658(1998)079[2032:rffatd]2.0.co;2.

Muñoz, A. E., \& Muñoz-Santibañez, P. (2016). Conflictos entre fauna silvestre y agricultura en Chile. Agronomía y forestal, 53, 10-17.

Nigussie, Z., Tsunekawa, A., Haregeweyn, N., Adgo, E., Cochrane, L., Floquet, A., \& Abele, S. (2017). Applying Ostrom's institutional analysis and development framework to soil and water conservation activities in northwestern Ethiopia. Land use policy, 71, 1-10. https://doi.org/10.1016/j.landusepol.2017.11.039.

North, D. (1990). Institutions, Institutional Change and Economic Performance. Cambridge. Cambridge University.

Ojasti, J., \& Dallmeier, F. (2000). Manejo de fauna silvestre neotropical. Smithsonian Institution/MAB Biodiversity Program, Washington D.C.

Ostrom, E. (1991). A Framework for Institutional Analysis. En Workshop in Political Theory and Policy Analysis. Indiana University.

Ostrom, E., Gardner, R., \& Walker, J. (1994). Rules, games, and common-pool resources. University of Michigan Press. https://doi.org/10.3998/mpub.9739.

Ostrom, E. (2000). El gobierno de los bienes comunes. La evolución de las instituciones de acción colectiva. México, D. F.: Fondo de Cultura Económica.

Ostrom, E. (2005). Understanding Institutional Diversity. Princeton University Press. 375 p.

Payán-Garrido, E., \& Soto-Vargas, C. (2012). Los Felinos de Colombia. Ministerio de Ambiente y Desarrollo Sostenible, Instituto de Investigaciones de Recursos Biológicos Alexander von Humboldt y Panthera Colombia. 48 pp.

Ciencia y Agricultura (Cien. Agri.) Vol. 16 (3). L-ISSN 0122-8420. elSSN 2539-0899.

Septiembre-Diciembre 2019, pp. 61-73. Tunja (Boyacá) - Colombia. DOI:

https://doi.org/10.19053/01228420.v16.n3.2019.9889 